Clinician's Guide to Nuclear Medicine. Gastroenterology L.K. Harding \& P.J.A. Robinson, Edinburgh: Churchill Livingstone, $1991,140 \mathrm{pp}$.

This small handbook is one of a series providing a clinician's guide to nuclear medicine. It reviews the whole area of gastroenterology including oesophageal and gastric dysmotility, malabsorption, liver and biliary disease, abdominal inflammation and sepsis and gastro-intestinal haemorrhage. Within each section there are brief details of the techniques used, clinical indications, what is involved for the patient including the radiation dose and interpretation of the results,

\section{Clinician's Guide to Nuclear Medicine. Benign and Malignant} Bone Disease

J.H. McKillop \& I. Fogelman, Edinburgh: Churchill Livingstone, 1991, $144 \mathrm{pp}$.

This small handbook is one of a series covering the clinical application of Nuclear Medicine techniques in specific fields. An introductory chapter briefly outlines the principles of bone scintigraphy including pharmacokinetics and imaging methodology. The widespread use of bone scanning in oncology for staging and detection of metastatic disease is fully discussed and a paediatric chapter includes a section on bone scintigraphy in childhood malignancy.

The remaining chapters covering bone and joint infection, metabolic bone disease, trauma, arthritis and avascular ne-

\section{Cancer and Aging}

Edited by A. Macieira-Coelho and B. Nordenskjöld, Bac Raton, Florida: CRC Press, 1991, 289 pp.

This book is meant to review the cancer-age relationship, both from the epidemiological, the experimental and the clinical evidence. The authors question the general idea of the potentiating effect of aging on cancer.

They postulate that cancerogenesis is a very complex and dynamic process, including many variables. The authors divide the material in 2 parts; the first deals with experimental evidence including developmental cell and tissue determinants; the second with cancer incidence and progression. The first part is well written and gives original ideas about cancer and aging at the cellular level. Much attention is given

\section{Tumours of the Newborn and Infant,}

H. Isaacs, Jnr., St. Louis: Mosby-Year Book Inc., 1991, 251 pp. $£ 53.50$.

This book represents an attempt by Hart Isaacs Jnr to demonstrate the 'gross pathologic and histopathologic features of neoplastic diseases and tumour-like conditions during the first year of life'. It is based largely upon the author's own experience over the last 27 years at the Children's Hospital of Los Angeles, with cross-references to studies from other centres.

The primary target for the publication is pathologists, with the specific aim being to help them interpret difficult histopathological appearances in developing, rather than mature tissues. However, it does include numerous case histories which increases its relevance to clinicians.

The first four chapters present a general background to tumours in infancy including aetiology, incidence/mortality, and diagnositic considerations. These are fairly succinct, although the incidence statistics could be clarified by using more uniform populations and comparable definitions. However, I must disagree with the author's unequivocal view that needle biopsy is contra-indicated in the initial diagnosis of paediatric solid neoplasm - 'since it is rare for enough tissue which is aided by extensive illustrations of high quality.

While radiotherapists and oncologists will rarely encounter most of the conditions described, this would be a useful handbook to be available for reference in specific circumstances including suspected bile leaks, malabsorption of vitamin B12 after lower abdominal radiotherapy, suspected intra-abdominal sepsis, gastrointestinal haemorrhage and the place of immunoscintigraphy in staging of malignancy and detection of tumour recurrence.

E.P. Wraight

crosis emphasise that bone scintigraphy has widespread clinical applications outside the oncological field. While these conditions may not often occur in radiotherapy and oncology clinical practice, awareness of other patterns of abnormality is important when interpreting scintigrams performed for the assessment of malignancy.

There are extensive illustrations of high quality although the more widespread use of arrows to draw attention to salient features would have been helpful.

This little handbook will be a useful source of reference for clinicians in general and for oncologists and radiotherapists in particular.

E.P. Wraight

to alterations in connective tissues and to the mechanism of metastasis. Two small chapters deal with effects of age on DNA-repair and immunity.

A large number of epidemiological and clinical subjects form the contents of the rest of this book. From these the reviewer found the chapter on progeroid syndromes and cancer of considerable interest. It is difficult to place chapters on familial colorectal cancer, late radiation effects and the development of the human breast in the context of this book. The chapters on the individual tumour types offer few new data to the general oncologist.

The experimental part of this book is excellent and it is therefore worthwhile to study it.

\section{F.J. Cleton}

to be obtained and it is possible to miss the diagnostically important area' (Plus 'formal exploratory operations have fewer serious immediate complications'). This view may have been sound many years ago, but CT and ultrasound guided biopsy is now so accurate, and so much less invasive than open biospy, that needle biopsy increasingly has a role in tissue diagnosis.

The subsequent sections on specific disease cover a wide range of material from specific organ-related tumours, such as renal and liver tumours through to more nebulous concepts such as soft tissue tumours and tumour-like conditions of the skin. Most of these are clearly written with excellent photographs, especially of the gross pathological specimens. Unfortunately some of the photomicrographs lose their impact by being printed in black and white, especially when the arrow markers are in black too.

The chapters on retinoblastoma and renal tumours are particularly notable for their clarity and depth, respectively. However, I fail to see how the section on neuroblastoma could include so little detail about stage $4 \mathrm{~S}$ neuroblastoma, which is by definition a 'tumour of infancy' (Also, no mention of deletion of the short arm of chromosome 1 as a prognostic indicator). Another contentious area deserving greater attention, is the supposed 'unifying concept' of primi- 
tive neuroectodermal tumours as a small cell malignant tumour of the central and peripheral nervous system.

Overall, despite the individual criticisms, this book provides a comprehensive review of a wide range of tumours in the very young child. It has been written by a single author, and therefore suffers from a certain degree of personal bias, especially regarding the clinical cases. However, this makes it much more readable from a clinicians viewpoint, and a valuable introduction to this complex and often confusing area.

P.Winyard 